\title{
Myoarchitecture and connective tissue in hearts with tricuspid atresia
}

\author{
D Sanchez-Quintana, V Climent, S Y Ho, R H Anderson
}

\begin{abstract}
Objective-To compare the atrial and ventricular myoarchitecture in the normal heart and the heart with tricuspid atresia, and to investigate changes in the three dimensional arrangement of collagen fibrils.

Methods-Blunt dissection and cell maceration with scanning electron microscopy were used to study the architecture of the atrial and ventricular musculature and the arrangement of collagen fibrils in three specimens with tricuspid atresia and six normal human hearts.

Results-There were significant modifications in the myoarchitecture of the right atrium and the left ventricle, both being noticeably hypertrophied. The middle layer of the ventricle in the abnormal hearts was thicker than in the normal hearts. The orientation of the superficial layer in the left ventricle in hearts with tricuspid atresia was irregular compared with the normal hearts. Scanning electron microscopy showed coarser endomysial sheaths and denser perimysial septa in hearts with tricuspid atresia than in normal hearts.

Conclusions-The overall architecture of the muscle fibres and its connective tissue matrix in hearts with tricuspid atresia differed from normal, probably reflecting modelling of the myocardium that is inherent to the malformation. This is in concordance with clinical observations showing deterioration in pump function of the dominant left ventricle from very early in life.

(Heart 1999;81:182-191)
\end{abstract}

Departamento de

Anatomia Humana,

Facultad de Medicina,

Universidad de

Exremadura, Badajoz,

Spain

D Sanchez-Quintana

V Climent

Imperial College School of Medicine, National Heart and

Lung Institute,

Dovehouse Street,

London SW3 6LY, UK

$S$ Y Ho

R H Anderson

Correspondence to: Dr Ho.

Accepted for publication 8 September 1998
Keywords: tricuspid atresia; congenital heart defects; connective tissue; fibrosis

The heart is a special type of pump that functions by means of a three dimensional arrangement of myocardial fibres supported by an extracellular collagenous matrix, the latter forming an intricate pattern within the ventricular wall. ${ }^{12}$ Over the past 300 years, different methods have been developed to study the organisation of myocardial fibres within the ventricular wall. These methods have been based either on gross dissection, ${ }^{3-5}$ or on a combination of dissection and histology. ${ }^{6}$ More recently, sophisticated methods based on three dimensional reconstruction from serial sections have been used. ${ }^{89}$ Nevertheless, a comparison of these various studies and methods has shown that some of the findings are controversial. ${ }^{10}$ One point of agreement is that interstitial collagen plays an important role in maintaining the size and shape of the heart. ${ }^{2}$ Ultrastructural studies have shown that this network consists of an organised hierarchy of collagen intimately associated with individual myocytes, with groups of muscle fibres, and with the coronary vasculature. ${ }^{11}$ Assignment of specific functions to one or more components of this network has not been fully achieved. There is evidence that in renovascular and genetic hypertension the hypertrophic response of the myocardium includes an increase in the concentration of collagen, with thickening of the existing fibrillar collagen. ${ }^{12}{ }^{13}$ Most studies of myocardial architecture have been confined to normal hearts, and few studies have compared dissections of normal and congenitally malformed hearts. ${ }^{14}{ }^{15}$ In the present study, we compared the atrial and ventricular myoarchitecture of the normal heart with that in a selected congenital malformation, tricuspid atresia. In addition to morphological observations, we used a cell maceration method ${ }^{16}$ to investigate the changes in the three dimensional arrangement of collagen fibrils.

\section{Methods}

We studied six specimens from patients with tricuspid atresia, comparing these with nine normal hearts. The specimens with tricuspid atresia were selected from the cardiopathological collection of the Royal Brompton Campus of the National Heart and Lung Institute, Imperial College, London. They came from patients ranging in age from 1 month to 8 years. The normal hearts came from subjects aged from 36 hours to 60 years old, all dying of causes unrelated to cardiac pathology. The normal hearts were fixed by immersion in $10 \%$ formaldehyde for several weeks before examination. The hearts from the cardiopathological collection had been fixed in $10 \%$ formaldehyde for many years, but their initial preparations were similar. They were initially fixed while intact by perfusion through the orifice of the inferior caval vein after ligation of other connecting vessels.

\section{DISSECTION}

Three specimens of tricuspid atresia and six normal hearts were used for gross dissection. The protocol used was similar to that followed in earlier studies, ${ }^{10}$ but on this occasion the atria were not removed, and at no time were the hearts boiled or enzymatically decollagenised. In studying the architecture of the myocardium, we use the term "muscle fibre" to describe a bundle of myocytes visible on gross inspection. 
Table 1 Layers of the left ventricle

\begin{tabular}{lllllll}
\hline Heart & Specimen & Age & $S L(\mathrm{~mm})$ & $M L(\mathrm{~mm})$ & $D L(\mathrm{~mm})$ & $\% M L$ \\
\hline 1 & TA & 1 month & 0.6 & 4.0 & 1.1 & 71 \\
2 & TA & 6 years & 0.9 & 9.0 & 1.5 & 78 \\
3 & TA & 8 years & 1.5 & 11.0 & 2.0 & 78 \\
4 & $\mathrm{~N}$ & 36 hours & 1.3 & 3.0 & 1.4 & 53 \\
5 & $\mathrm{~N}$ & 2 months & 1.6 & 4.0 & 1.5 & 56 \\
6 & $\mathrm{~N}$ & 4 years & 1.9 & 4.8 & 1.6 & 58 \\
7 & $\mathrm{~N}$ & 10 years & 1.9 & 5.1 & 1.7 & 58 \\
8 & $\mathrm{~N}$ & 32 years & 5.5 & 12.2 & 3.8 & 57 \\
9 & $\mathrm{~N}$ & 60 years & 6.0 & 13.0 & 4.0 & 56 \\
\hline
\end{tabular}

DL, thickness of deep layer; ML, thickness of middle layer; N, normal heart; SL, thickness of superficial layer; TA, tricuspid atresia; \%ML, thickness of the middle layer as percentage of total.

For the ventricles, the first stage of dissection was to remove carefully, with watchmakers' tweezers, the epicardium and coronary vessels without detaching muscle fibres from the subepicardial layer.

The second stage involved stepwise removal of muscle fibres, beginning superficially and working towards the ventricular cavities, documenting each step with serial photography. It is always possible with this technique to establish the grain of muscle fibres within the heart. There is always a major alignment of muscle fibres in the ventricular wall. Fibres parallel to the long axis of the ventricular mass are described as longitudinal, whereas fibres parallel to the short axis are described as circumferential. Fibres at an angle to these major axes are described as oblique. A change in orientation was taken to indicate the start of a different myocardial layer.

During dissection, the thickness of each layer was measured with a slide gauge at the level of the equator of the left ventricle (table 1). Since the atrial walls were thinner, removal of the epicardium and endocardium was adequate to reveal the arrangement of the myocardial fibres.

\section{CELL MACERATION/SCANNING ELECTRON}

MICROSCOPY

Three normal and three abnormal hearts from children of different ages (normal: 1 month, 4 months, 5 years; tricuspid atresia: 2 months, 4 months, 2 years) were used for scanning electron microscopy, after preparation involving a variation of the cell maceration method of Ohtani et al. ${ }^{16}$ Small tissue blocks containing myocardium of the left ventricle (approximately $7 \times 7 \times 7 \mathrm{~mm}$ ) were immersed in a $10 \%$ aqueous solution of sodium hydroxide for four days at room temperature. They were then placed in a $1 \%$ aqueous solution of tannic acid for three hours, rinsed in distilled water for seven hours, and postfixed in a $1 \%$ aqueous solution of osmium tetroxide for three hours. The specimens were dehydrated in a series of graded concentrations of acetone, freeze fractured with a razor blade in liquid nitrogen, and
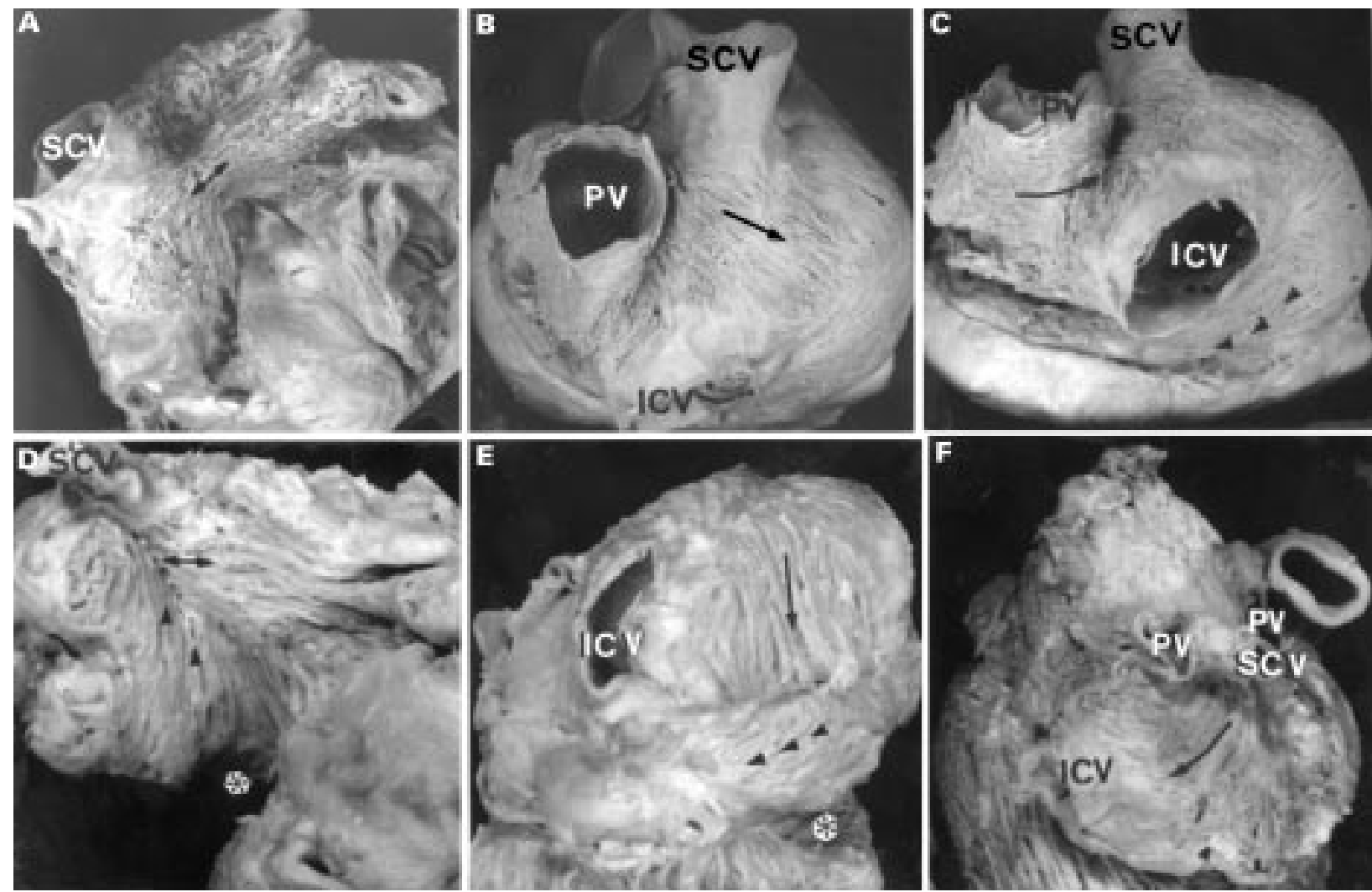

Figure 1 External views of the normal atria $(A, B, C)$ and tricuspid atresia specimens $(D, E, F)$, showing the arrangement of the subepicardial fibres on anterior $(A, D)$ and posterior sectors $(B, C, E, F)$. Note in panel $A$, Bachmann's bundle (double arrows) coursing horizontally from the cavoatrial junction to the left appendage. In contrast, in panel D the fibres of Bachmann's bundle tend to be aligned obliquely meeting with the fibres from the floor of the right atrium (arrowheads). In the posterior sector note (panel B) an intercaval bundle with a component of fibres obliquely from left to right (arrow) across the terminal groove, and an another component running obliquely from right to left (arrowheads in panel C) covered the dorsal wall of the coronary sinus. On the left atrium the fibres run circumferentially (arrow) into the posterior interatrial groove (panel $C$ ) and pass anteriorly to join the interatrial band. In contrast, in the specimens with tricuspid atresia, the intercaval bundle shows a preferentially longitudinal direction between the caval veins (arrow, panels $E$ and $F$ ) and a horizontal component in the floor of the right atrium (arrowheads, panel $E$ ). In the left atrium the fibres were arranged irregularly without one clear direction. A, aorta; ICV, inferior caval vein; LA, left appendage; PV, right pulmonary veins; $R A$, right appendage; SVC, superior caval vein. *Absence of right atrioventricular connection. 

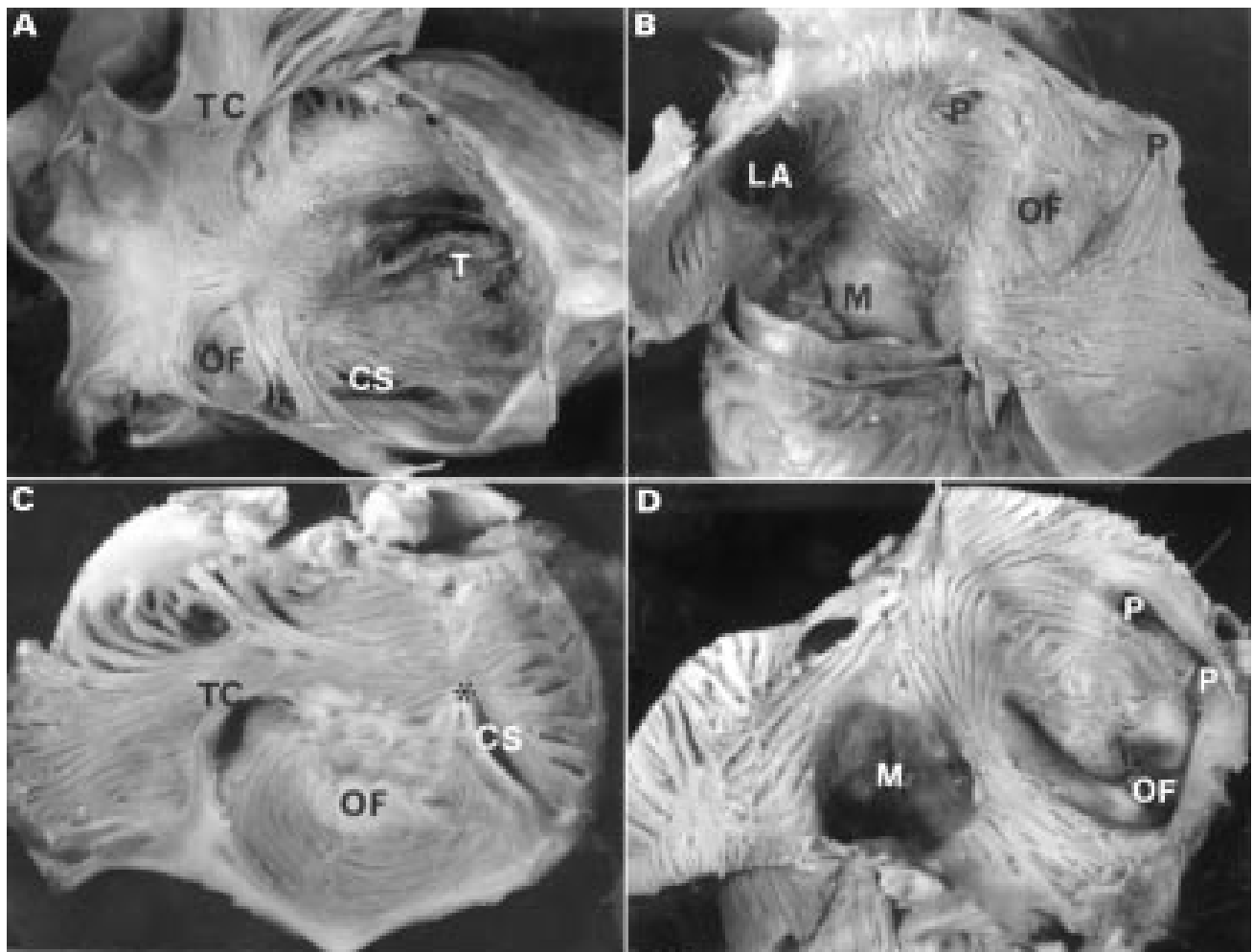

Figure 2 Internal view of the right atrium $(A, C)$ and left atrium $(B, D)$ after removal of the endocardium. Note in the normal right atrium (panel $A$ ) the arrangement of the fibres is distinctly different from that in the tricuspid atresia specimen (panel C). On the inner surface of the normal left atrium (panel B) fibres encircle the oval fossa, pulmonary veins, and the atrial appendage. Note in tricuspid atresia (panel D) there is a prominent circular pattern around the oval fossa and fewer longitudinal fibres toward the mitral valve. CS, orifice of the coronary sinus; LA, orifice of the left appendage; $M$, mitral valve; OF, oval fossa; $P$, orifices of pulmonary veins; $T$, tricuspid valve; TC, terminal crest. ${ }^{\star}$ Dimple.

dried to the critical point. The dried specimens were mounted on metal stubs, coated with gold, and observed under a scanning electron microscope.

\section{Results}

The typical external and internal morphological features of the normal hearts were altered in the three hearts with tricuspid atresia, dissected to display the orientation of the muscle fibres. In all these cases, the right atrioventricular junction and connection was absent, and the only exit for right atrial flow was through a septal defect within the oval fossa. Two cases showed right sided rudimentary right ventricles and discordant ventriculo-arterial connections. The right ventricle, lacking its inlet component, communicated with the dominant left ventricle through a muscular ventricular septal defect. In these two cases, there was obvious left ventricular hypertrophy, both margins of the ventricular mass being obtuse, and there was no evidence of interventricular grooves. The tip of the heart in both cases was formed only by the rounded apex of the left ventricle. Such morphological features produced a saccular or spherical shape to the heart. In the third case, the ventriculo-arterial connections were concordant. This heart had the largest right ventricle, although it still lacked its inlet component, which communicated with the left ventricle through a non-restrictive muscular ventricular septal defect. In this case, the interventricular grooves and margins on both the right and left sides were more evident, but left ventricular hypertrophy produced a distinctive cone shaped heart with a pointed apex.

MYOARCHITECTURE OF THE ATRIA

The normal atrial walls were composed of compact myocardium of profoundly varying thickness. In the right atrium, the thickest myocardium was at the terminal crest and the anterior wall of the appendage. The thinnest parts were at the vestibule and between the pectinate muscles. In the left atrium, the myocardium was more uniform, and the walls generally were thicker than those of the right atrium. The thinner parts were located posteriorly between the orifices of the pulmonary veins. In comparison with normal hearts, different features were seen in the atrial walls of the specimens with tricuspid atresia. The myocardium of the right atrium was, on average, twice the thickness of the left atrium.

OUTER (SUBEPICARDIAL) SURFACE

In the normal heart, two sectors, anterior and posterior, could be distinguished according to the arrangement of the myocardial bundles. The anterior sector encompassed the anterior and lateral aspects of the atria and the so called interatrial band. The subepicardial fibres in the normal atrium showed a horizontal arrangement, coursing from the superior cavoatrial junction to the left appendage and crossing in the anterior interatrial groove (Bachmann's bundle). On reaching the left appendage, the fibres divided into upper and lower branches 

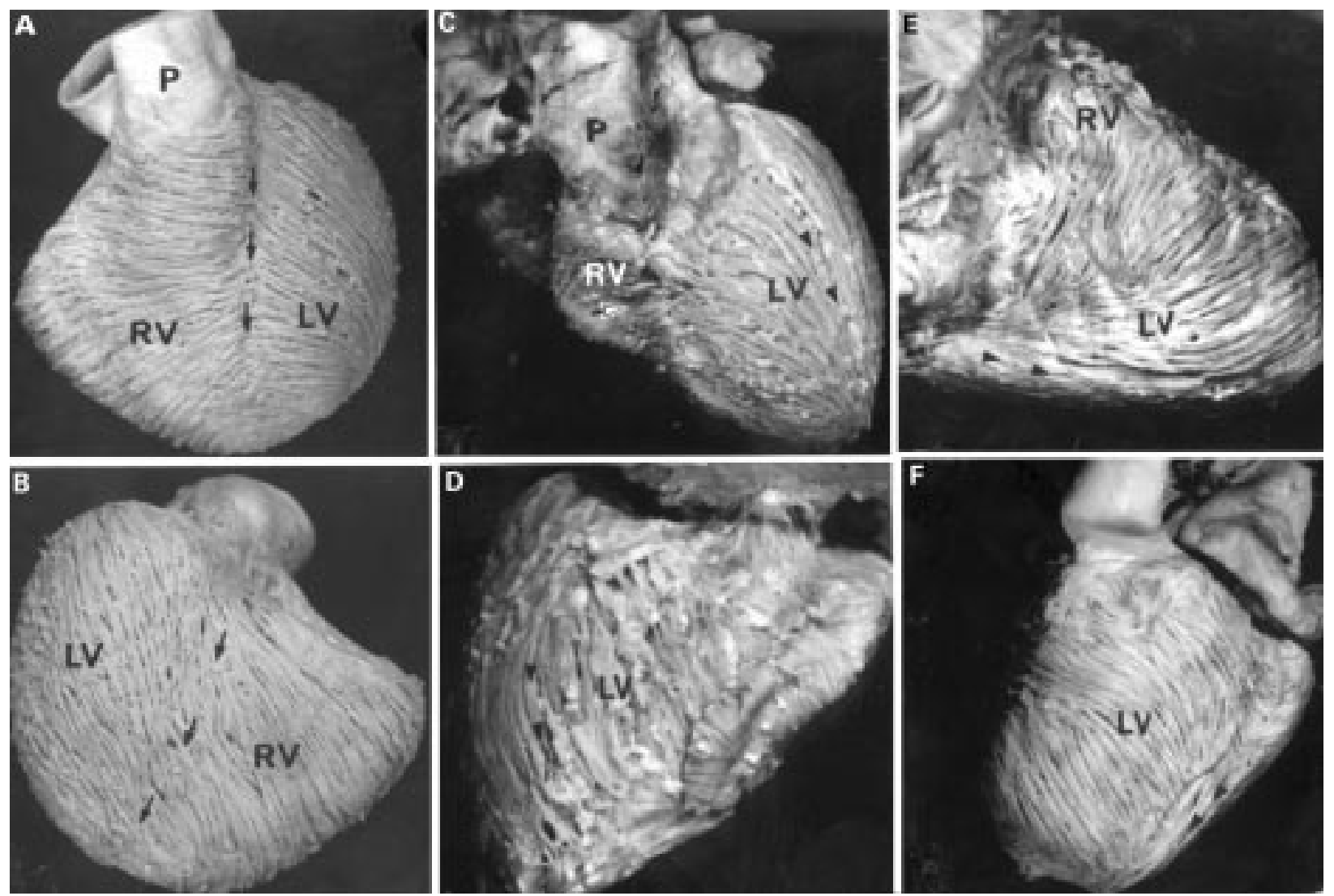

Figure 3 Views of the hearts of normal $(A, B)$ and tricuspid atresia specimens $(C, D, E, F)$, showing the arrangement of the ventricular fibres of the superficial layer. In panels $A$ and $B$ the fibres run obliquely across the interventricular grooves (arrows), although the posterior interventricular groove is less evident. The corresponding sternocostal surface (panel $C$ ) and diaphragmatic surface (panel D) of the heart with tricuspid atresia from a patient aged 8 years show lack of interventricular grooves. Note the longitudinal fibres (arrowheads) in the left ventricle. Panels $E$ and $F$ show right and left margins respectively of a heart from a one month old patient. The right ventricle is hypoplastic whereas the left ventricle is profoundly hypertrophied in this case. Note the longitudinal fibres (arrowheads) on the diaphragmatic surface. A, aorta; LV, left ventricle; P, pulmonary trunk; RV, right ventricle. Open arrow, horizontal fibres; arrowheads, longitudinal fibres.

which encircled the appendage and subsequently rejoined in the posterior sector (fig 1A) which covered the posterior aspect of the normal atria. An intercaval bundle, with oblique fibres, passed between the orifices of the caval veins, crossed the terminal groove, and continued into the pectinate muscles of the right atrium (fig 1B). Another set of oblique fibres covered the posterior wall of the coronary sinus and the anterior margin of the inferior caval vein, terminating in the interatrial septum (fig 1C). In addition, another set of horizontally arranged muscle fibres passed from the posterior interatrial groove to the left atrium. These ran to the mitral vestibule and continued superiorly, between the orifices of the pulmonary veins, where they intermingled with fibres from the interatrial band (fig 1C). At the level of the pulmonary veins, these fibres divided into two groups which encircled each orifice.

Variations in arrangement of the fibres were observed in the hearts with tricuspid atresia, in large part because of the absence of the atrioventricular connection. The fibres of the interatrial band were aligned more obliquely and met with the fibres from the floor of the right atrium (fig 1D). Only a small number of these fibres from the interatrial band turned to the posterior sector (fig 1D). The intercaval bundle was thicker than in the normal heart. This fascicle contained a longitudinal compo- nent of fibres which ran throughout the intercaval area (fig $1 \mathrm{E}, \mathrm{F}$ ), together with a horizontal component of fibres which covered the underside of the floor of the right atrium (the putative tricuspid vestibule) (fig $1 \mathrm{E}$ ). At the posterior surface of the left atrium, the fibres were arranged irregularly, intermingling horizontal with oblique fibres (fig $1 \mathrm{~F}$ ).

INNER (SUBENDOCARDIAL) SURFACE

In the normal right atrium, the myocardial fibres from the terminal crest ran longitudinally through the pectinate muscles, extending anteriorly and laterally to the atrial appendage, and medially to the septal wall along the rim of the oval fossa. At the inferior part, thin fibres encircled the orifice of the coronary sinus, and other fibres passed to the tricuspid vestibule (fig 2A). In the normal left atrium, the fibres were arranged in loops around the mitral vestibule, oval fossa and pulmonary veins. Circumferential fibres were limited to the level of the flap valve of the oval fossa, being most obviously seen where its two horns mark the usual site of fusion with the rim (fig 2B).

In contrast to the normal hearts, all the specimens with tricuspid atresia showed complete absence of the tricuspid valve and the right atrioventricular vestibule. The subendocardial fibres were arranged radially from the muscular floor, or dimple, toward the atrial 

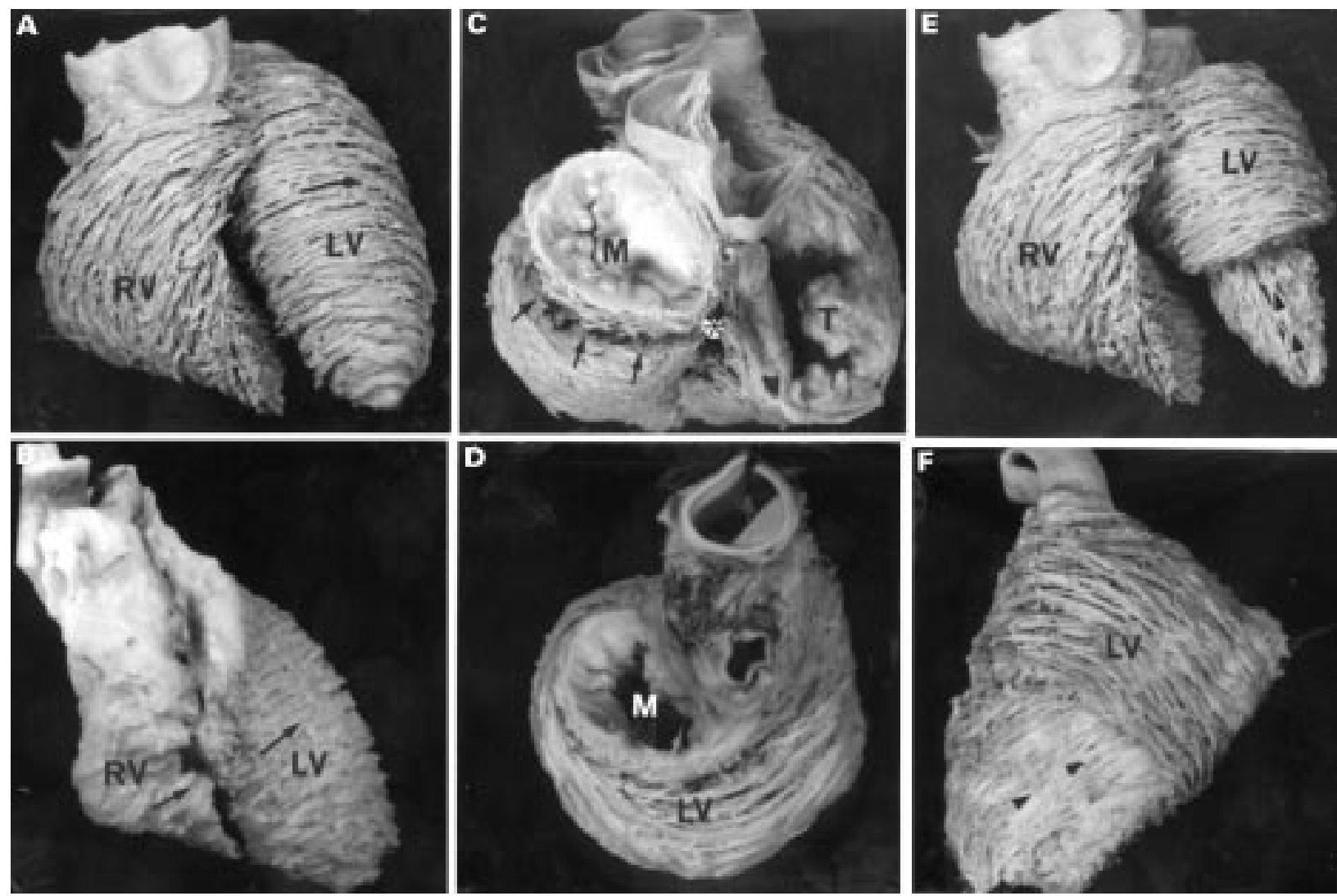

Figure 4 Views of the normal heart $(A, C, E)$ and tricuspid atresia specimens $(B, D, F)$, showing the arrangement of the ventricular fibres in the middle $(A-D)$ and deep layers $(E, F)$. The sternocostal aspects (panels $A$ and $E$ ) show the circular arrangement (arrow) of the left ventricular fibres, in contrast to the oblique arrangement of the right ventricular fibres in the normal heart. A comparable view of the abnormal heart (panel B) shows circular fibres in both ventricles (arrows). The basal views (panels $C$ and D) show the distinct invagination at the crux (asterix) and the basal aperture (arrows) in the normal heart, whereas the left ventricle predominates in the abnormal heart where there is no evidence whatever of the tricuspid valve (panel D). The deep layers (arrowheads) are revealed by removing the apical half of the middle layer (panels $E$ and $F$ ). The heart with tricuspid atresia is rotated to show its left (obtuse) margin. A, aorta; LV, left ventricle; $M$, mitral valve; $P$, pulmonary trunk; RV, right ventricle; $T$, tricuspid valve.

walls, with the exception of the atrial septum where they made a noticeably circular pattern around the rim of the oval fossa (fig $2 \mathrm{C}$ ). The fibres on the inner surface of the left atrium were similar to those seen in the normal hearts. In the specimen from the youngest child, nonetheless, there was a predominantly circular arrangement around the oval fossa, and there were fewer longitudinal fibres passing into the mitral vestibule (fig 2D).

MYOARCHITECTURE OF THE VENTRICLES

In both normal and abnormal hearts, the ventricular mass was composed of three layers of muscle fibres: superficial (subepicardial), middle, and deep (subendocardial). As indicated, the distinction between one layer and the next was made on the basis of a change in the orientation of the muscle fibres through the ventricular wall. We were not able to distinguish discrete planes of cleavage, or fibrous septa, between the three layers. The superficial and deep layers were present in both ventricles. The middle layer was present only in the left ventricle.

In the normal heart, the thickness of the muscular mass of the ventricles depended of the age of the individual. The normal middle layer occupied $53-59 \%$ of the ventricular wall thickness, while the superficial layer occupied $25 \%$, the rest being the deep layer (table 1). The relative thickness of each layer was almost constant. In striking contrast to the normal heart, the middle layer in all hearts with tricuspid atresia was thicker, making up about $71-78 \%$ of the total ventricular wall (table 1). The superficial layer was thinnest and, in the youngest specimen, constituted a very thin sheath.

\section{Superficial layer}

The superficial or subepicardial layer in the normal hearts consisted of muscle fibres which passed from the base of the heart toward the apex, extending from one ventricle to the other to form one common layer. At the apex, the superficial layer invaginated in a spiral pattern to continue into the deep, or subendocardial, layer. The fibres on the sternocostal aspect ran obliquely, crossing the anterior interventricular groove (fig 3A), while on the diaphragmatic aspect they crossed the posterior interventricular groove (fig 3B). The fibres in the superficial layer of the right ventricle were arranged more circumferentially than in the left ventricle.

Very different arrangements were seen in the hearts with tricuspid atresia. These modifications depended on the degree of underdevelopment of the rudimentary right ventricle, and on the ventriculo-arterial connections. When the right ventricle was larger-in the conical heart with concordant ventriculo-arterial connections - the fibres on the sternocostal aspect were still circumferentially arranged in 


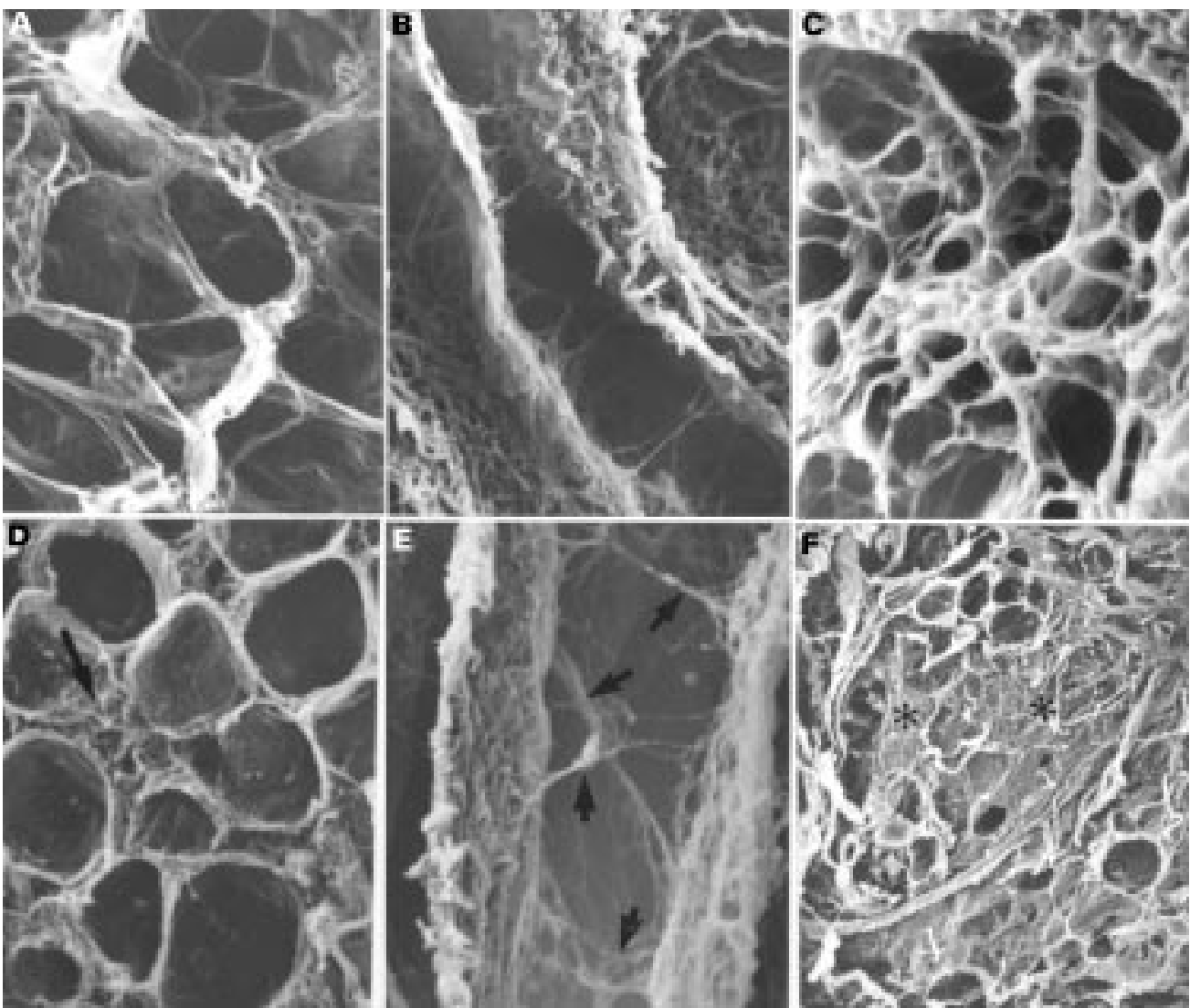

Figure 5 Scanning electron micrographs show the spatial arrangement of the endomysial component after $\mathrm{NaOH}$ digestion of the myocardial cells in the left ventricle of the normal heart $(A, B, C)$ and tricuspid atresia specimen $(D, E, F)$. Note in panel $D$ the disrupted endomysial sheath (arrow), in panel $E$ the network of struts (arrows), and in panel $F$ the areas of interstitial fibrosis (asterisks). Magnifications: panels $A$ and $D, \times 392$; panels $B$ and $E, \times 522 ;$ panels $C$ and $F, \times 94$. Normal: 1 month; tricuspid atresia: 2 months.

the right ventricle but turned obliquely in the left ventricle. They were strikingly longitudinal in the obtuse margin (fig $3 \mathrm{C}, \mathrm{D}$ ). When the rudimentary right ventricle was severely hypoplastic in the setting of discordant ventriculoarterial connections, the heart lacked interventricular grooves. The fibres on the sternocostal aspect ran horizontally (circumferentially) in the right ventricle, but were irregularly oriented in the left ventricle in comparison with the normal heart. They showed a circumferential orientation in the basal portion of the obtuse margin, an oblique orientation in the apical portion, and a longitudinal arrangement on the diaphragmatic aspect.

\section{Middle layer}

In normal hearts, the left ventricle contained a middle layer of myocardial fibres, the fibres being oriented more horizontally than those of the superficial layer (fig 4A). This layer was thickest at the equator, thinning out towards both the basal and apical ends. At the apical end, the middle layer enclosed a small aperture through which the superficial muscle fibres invaginated to become subendocardial. In the basal area, the middle layer formed large oval aperture because it did not terminate at the aortic-mitral unit (fig 4C). No proper middle layer could be defined in the normal right ventricle, no changes being seen in the orientation of the fibres in the mid-portion of the ventricular wall (fig 4A).

In all cases with tricuspid atresia, the middle layer of the left ventricle was noticeably thicker than in the normal hearts, conferring a circumferential arrangement (fig 4B, D). Since the superficial fibres in the rudimentary right ventricle were already horizontally aligned, no change in orientation was found to signify the presence of a middle layer, as the circumferential fibres of the left ventricle continued into the right ventricle (fig $4 \mathrm{~B}, \mathrm{D}$ ).

\section{Deep layer}

In the normal hearts, the deep (or subendocardial) layer was composed of preferentially arranged longitudinal fibres which passed through the vortices toward the papillary muscles, to the atrioventricular orifices and the arterial orifices, and to the ventricular septum (fig 4E).

Several differences were seen in the hearts with tricuspid atresia. In all cases, papillary muscles were absent from the right ventricle, so the fibres that invaginated at the right vortex were confined to the apical trabecular component. They were irregularly oriented, showing longitudinal fibres intermingled with circumferential fibres. In the left ventricle, in contrast, the fibres from the left vortex were arranged obliquely, and lined the ventricular cavity to spiral toward the mitral valve (fig $4 \mathrm{~F}$ ), but ran 

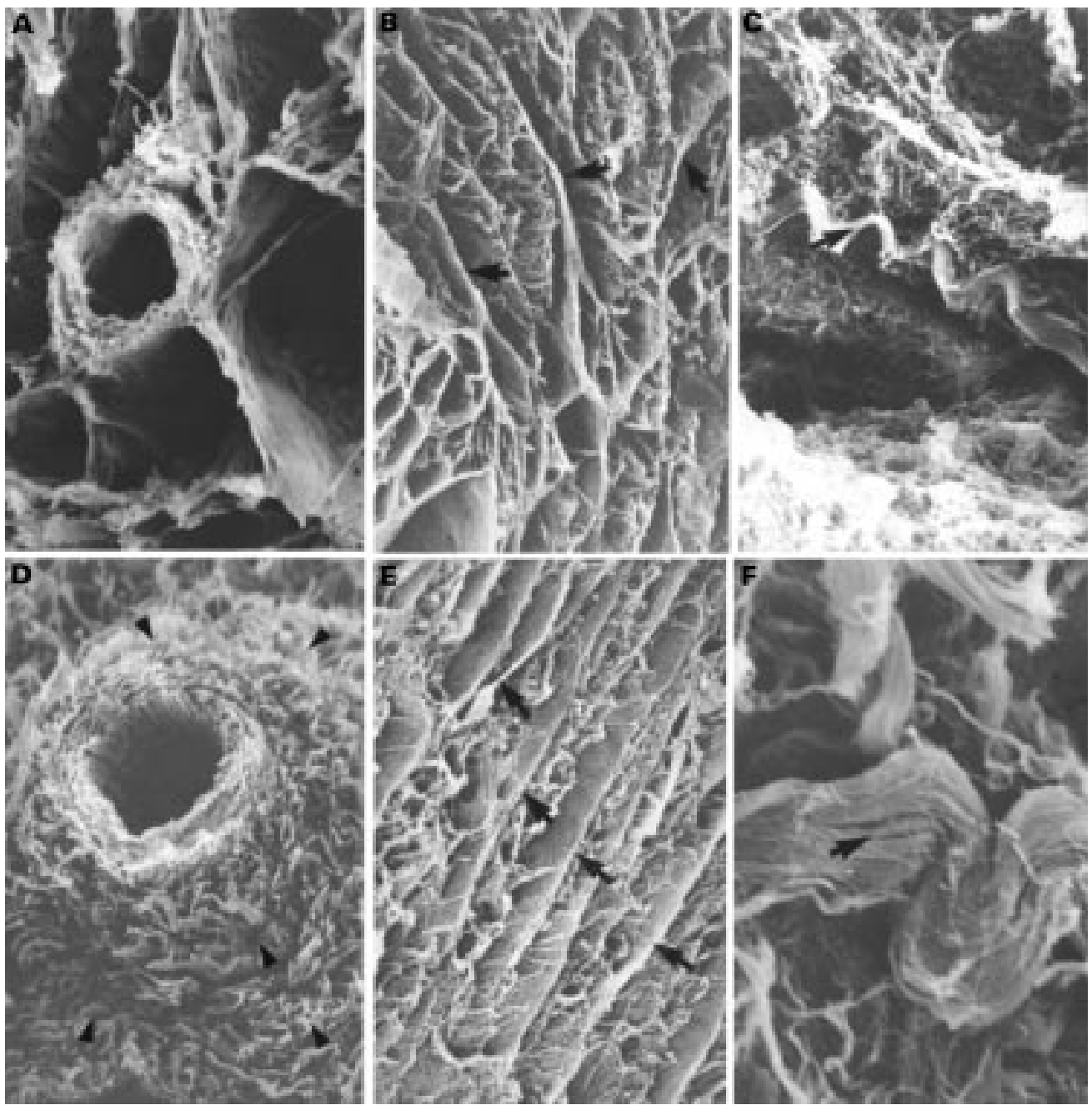

Figure 6 Scanning electron micrographs showing the spatial arrangement of the perimysial component around the blood vessel in a normal heart (A) and with perivascular fibrosis (arrowheads) in tricuspid atresia specimen (D). Structurally the perimysial component in the normal heart $(B, C)$ and tricuspid atresia specimen $(E, F)$ is composed of septa that separate muscle bundles $(B, E)$ and spiral tracts (coiled perimysial fibres) (arrows) that run obliquely or parallel to the septa $(C, F)$. The heart with tricuspid atresia show more numerous perimysial septa (arrows, panel E) and coarser spiral tracts (arrow, panel F). Magnifications: panels $A$ and $D, \times 261$; panels $B$ and $E, \times 52$; panel $C, \times 313$; panel $F, \times 522$.

Normal: 4 months; tricuspid atresia: 4 months.

longitudinally in the papillary muscles. When the fibres reached the ventricular septal defect, they surrounded it and were closely related to the leaflets of the arterial valves.

ARCHITECTURE OF THE CONNECTIVE TISSUE

The three dimensional architecture of the intramyocardial connective tissue in different zones of the normal and abnormal ventricular myocardium was examined following removal of cellular components. We distinguished three components of the connective tissue matrixfirst, the endomysium around individual myocytes; second, the perimysium around groups of myocardial cells; and third, the epimysium, which ensheathed the whole of the myocardium and separated it from the epicardium and endocardium. The scanning electron micrographs revealed several differences in the matrix between normal and malformed hearts. These modifications related to the presence of left ventricular hypertrophy, but not to the type of the ventriculo-arterial connections or the age of the specimens.
In normal myocardium, the endomysium showed a honeycomb appearance where the cavities varied in size and consisted of fine and smooth sheaths of collagen fibres arranged around the myocytes (fig 5A, C). Each sheath was connected to its neighbour by struts, which were thin collagen tracts arranged as bridges between the sheaths (fig 5B). In contrast, the endomysial sheaths in hearts with tricuspid atresia were much coarser (fig 5D), with the struts appearing more numerous, sometimes forming a complex network in the gaps between the myocytes (fig $5 \mathrm{E}$ ). Furthermore, occasional endomysial sheaths were disrupted, and various amounts of collagen fibres were accumulated between groups of myocytes (interstitial fibrosis) (fig $5 \mathrm{~F}$ ). Accumulations of fibrillar tracts of the endomysial or perimysial components were also more prominent around the small capillaries or arterioles and venules, producing perivascular fibrosis in the malformed hearts (compare with fig 6A, D).

In normal myocardium, the perimysium was composed of a dense network of reticular and 

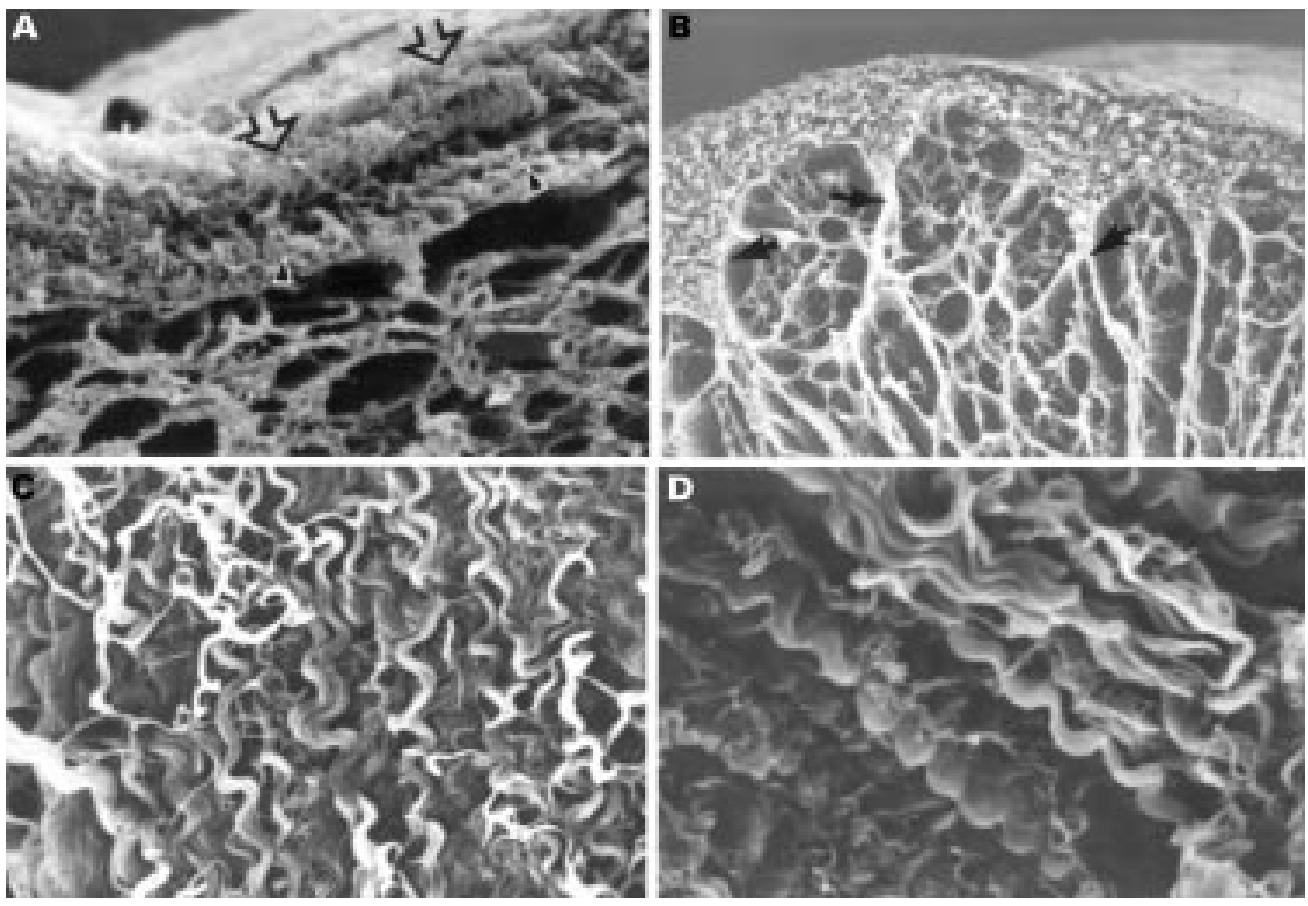

Figure 7 Scanning electron micrographs showing the epimysial component in normal $(A, B, C)$ and tricuspid atresia specimens (D). The outer layer of the epimysium (panel $A$ ) has many spiral fibres (panel $C$ ) between epicardium (open arrow) and the inner layer of epimysium (arrowhead). The inner layer is continuous with perimysial septa (arrows, panel $B)$. In tricuspid atresia specimens the spiral tracts were coarser than in normal hearts (panel D). Magnifications: panel A, $\times 73$; panel B, $\times 52$; panels $C$ and D, $\times 311$. Normal, panel A: 5 years; panels $B$ and $C: 1$ month; tricuspid atresia: 2 years.

collagen fibrils running in various directions between groups of myocytes (fig 6B). Adjacent, and arranged parallel to the perimysium, were coiled tendon-like perimysial fibres, which were formed by bundles of collagen fibres (fig 6C). Considerable variation in the architectural pattern was seen in the myocardium in tricuspid atresia. There were more perimysial septa, even in the youngest heart (fig 6E). More collagen bundles were found in each coiled perimysium and, as a result, these coiled fibres were coarser than in normal hearts (fig 6F).

In normal hearts, the epimysium is a structure of varying thicknesses composed of two distinct layers of sheaths (fig 7A). The outer layer of epimysium consisted of collagen fibres arranged in a regular wavy pattern between the pericardium, or epicardium, and the inner layer of the epimysium (fig 7C). The inner epimysial layer was composed of a coarse sheath formed by a complex array of collagen and reticular fibres that surrounded the myocardium (fig 7A, B). The perimysial septa arising from the inner sheath arborised in the thickness of the myocardium to form the perimysium (fig 7B). The arrangement of the epimysium within the myocardium in tricuspid atresia was similar to that in normal heart. The outer layer of the epimysium, however, contained fewer collagen fibres immediately beneath the pericardium, but they were coarser than in normal hearts (fig 7D).

\section{Discussion}

Our results show notable morphological changes in atrial and ventricular myoarchitecture in tricuspid atresia in comparison with the normal heart. Previous studies in the normal heart have shown that the atrial walls are composed of circumferential and longitudinal fibres. The arrangement of the fibres correlates well with known patterns of atrial contraction. ${ }^{17}$ The interatrial band probably accounts for the reduction in the anteroposterior dimension of the left atrium observed during left atrial systole. ${ }^{18}$ In contrast, the longitudinal fibres are positioned to produce upward motion of the atrioventricular junction, which is also known to occur during atrial systole. ${ }^{18}$ The reduction in longitudinal fibres in one of the abnormal hearts could have implications for motion of the atrioventricular junction. Several studies have also shown that the mode of conduction of impulse within the muscular walls of the atria reflects the arrangement of well developed muscular bundles, although it must be remembered that these bundles are not insulated electrically from the rest of the atrial myocardium. ${ }^{19} 20$

Most studies of the gross anatomy with tricuspid atresia have focused on the morphological and echocardiographic features of the ventricles and great vessels. Relatively little attention has been paid to the functional role that alterations in atrial or ventricular myoarchitecture might play in the clinical setting. ${ }^{21}$ Our findings reveal significant modifications in the myoarchitecture of the right atrium in tricuspid atresia. This is hardly surprising, since the morphological substrate for the atresia in almost all cases is absence of the entire right atrioventricular connection, including the tricuspid valve and its vestibule. The lesion is only very rarely due to an imperforate atrioventricular valve. ${ }^{22}$ Our dissections 
are unequivocal in showing the absence of the right atrioventricular connections.

In our normal hearts, we confirmed the typical pattern of ventricular myoarchitecture shown in our previous studies. ${ }^{610}$ In the left ventricle, the characteristic pattern is one of three concentric layers, with myocardial fibres extending through the ventricular wall in subepicardial (or superficial), middle, and subendocardial (or deep) locations. ${ }^{6}{ }^{1014}{ }^{15}$ In contrast, only superficial and deep layers are found within the wall of the normal right ventricle. These findings are very different from the descriptions of Torrent-Guasp, ${ }^{5}$ who suggested that the ventricles are made up of a solitary system of fibres which can be traced from the aortic to the pulmonary roots. These interpretations reflect the way in which the heart is opened by Torrent-Guasp. ${ }^{5}$ As a consequence of the absence of one atrioventricular connection in tricuspid atresia, the ventricular myoarchitecture is obviously different from normal. The arrangement of the rudimentary and incomplete right ventricle is then further modified by the ventriculoarterial connections. The overall architecture is still further influenced by the presence of left ventricular hypertrophy, the thickness of the middle layer far exceeding that of normal hearts in all hearts studied. Despite the small number of cases, we also observed obvious differences in the pattern of arrangement of the superficial layer and in the shape of the heart when compared with normal hearts. Thus the superficial fibres of the right ventricle are more horizontal than in the normal heart. In the solitary case with concordant ventriculo-arterial connections, the superficial fibres of the left ventricle turned noticeably longitudinal in the left ventricle, especially along the obtuse margin. In the remaining two cases, the fibres were irregularly oriented, without the smooth transitions seen in the normal heart.

Early studies reported that, even when the left ventricle was hypertrophied, the fibres showed the same orientation as in the normal heart. ${ }^{23}$ Pearlman et $a l,{ }^{24}$ however, found a predominance of circumferentially oriented fibres at the equator of the hypertrophied but normally structured left ventricle. They argued that this may reflect adaptation of the muscular architecture to support the greater circumferential stress that exists in the myocardium as a result of the approximately ellipsoidal shape of the left ventricle. ${ }^{25}$ The finding of an equally hypertrophied middle layer in our youngest specimen (1 month), however, suggests that this feature is inherent to the malformation itself. In the absence of any inlet component within the right ventricle, the relation between the ventricles in tricuspid atresia must be different from normal. These anatomical observations are in concordance with clinical observations that the shape and ejection fraction of the left ventricle is frequently abnormal, from the earliest ages. ${ }^{26}$ The regional differences in alignment of the fibres in the superficial layer could well account for disturbances in wall movement observed in clinical studies. ${ }^{28}$
We used scanning electron microscopy to demonstrate the well organised hierarchical extracellular network of collagen, since much of the fibrillar component cannot be seen by light microscopy. ${ }^{11}$ Collagen is known to be a major component of the intramyocardial connective tissue matrix. ${ }^{29}$ In our specimens of tricuspid atresia, observed by scanning electron microscopy, the connective tissue largely had a fibrillar appearance. We presume from this that the increase in connective tissue of the malformed heart is primarily caused by an increase in collagen. In our previous study, ${ }^{30}$ we observed an increase in fibrous content in both right and left ventricles in tricuspid atresia. Our present study shows that, in addition to these quantitative increases and unrelated to the type of ventriculoarterial connections, there are morphological changes in the arrangement of the intramyocardial connective tissue, accompanied by remodelling of the collagen matrix. This remodelling involves the endomysium, perimysium, and the epimysium. We observed a thickening of collagen struts, fibrils, and coiled perimysial fibres, an increase in the density of the endomysial sheaths surrounding the myocytes, an increase in the number of perimysial septa, an expansion of the area occupied by perivascular collagen, microscopic damage of endomysial sheaths, and microscopic scars. The remodelled architecture must surely contribute to the functional abnormalities of the malformed hearts. It is conceivable that the arrangement of the fibrous septa greatly reduces both the contractility and conduction of the myocytes through the loss of cell to cell contacts. The cause of the increase in connective tissue, however, remains uncertain. Although it has been argued that hypertrophy of muscle fibres and increased collagen content are independently regulated, ${ }^{31}$ this viewpoint is not widely held. Another view is that synthesis of collagen is related to wall stress. An alteration in the arrangement of collagen could either be a response to an increase in force or to an altered distribution of force, to coordinate its transmission to the ventricles. ${ }^{32}{ }^{33}$ Although we cannot prove or disprove these hypothesis by morphological studies, our results suggest that the changes in intramyocardial connective tissue and myoarchitecture could be considered an additional factor of the abnormal development in tricuspid atresia. We still do not know at which developmental stage it occurred.

\section{LIMITATIONS OF STUDY}

We were limited by having only a few specimens available for study. Although larger numbers are desirable, we are responsible for maintaining and preserving the specimens in our collection for teaching purposes. We are reluctant to sacrifice more specimens for this totally destructive method of investigation but hope that this study will stimulate other investigators with available material to emulate these studies.

During this investigation DS-Q was supported by the grant DGICYT (PR95-057) from the Spanish government. SYH and RHA are supported by the British Heart Foundation, together 
with the Joseph Levy Foundation. This study was funded by British Heart Foundation project grant PG 93060.

1 Streeter DD. Gross morphology and fiber geometry of the heart. In: Berne RM, Sperelakis N, Geiger SR, eds. Handbook of physiology. The cardiovascular system. Baltimore: Wilbook of physiology. The cardiovascular
liams and Wilkins, 1979:61-112.

2 Caulfield JB, Janicki JS. Structure and function of myocardial fibrillar collagen. Technol Health Care 1997;5 95-113.

3 Mall FP. On the muscular architecture of the ventricles of the human heart. Am $\mathcal{F}$ Anat 1911;11:211-66.

4 Grant PP. Notes on the muscular architecture of the left ventricle. Circulation 1965;32:301-8.

5 Torrent-Guasp F. La estructura macroscopica del miocardio ventricular. Rev Esp Cardiol 1980; 33:265-87.

6 Greenbaum RA, Ho SY, Gibson DG, et al. Left ventricular fibre architecture in man. Br Heart f 1981;54:248-63.

7 Fernandez-Teran MA, Hurle JM. Myocardial fiber architecture of the human heart ventricles. Anat Rec 1982;204:137ture

8 McLean MR, Prothero J. Coordinated three-dimensional reconstruction from serial sections at macroscopic and
microscopic levels of resolution: the human heart. Anat Rec microscopic levels

9 Jouk PS, Usson Y, Michalowicz G, et al. Mapping of the orientation of myocardial cells by means of polarized light and confocal laser microscopy. Microsc Res Tech 1995;30:48090.

10 Sanchez-Quintana D, Garcia-Martinez V, Climent V, et al. Morphological changes in the normal pattern of ventricular myoarchitecture in the developing human heart. Anat Rec 1995;243:483-95.

11 Caulfield JB, Borg TK. The collagen network of the heart. Lab Invest 1979;40:364-72.

12 Holubarsch CH, Holubarsch T, Jacob R, et al. Passive elastic properties of myocardium in different models and stages of hypertrophy: a study comparing mechanical, chemical, and morphometric parameters. Perspect Cardiovasc Res 1983;7:323-36.

13 Weber KT, Brilla CG. Pathologic hypertrophy and cardiac interstitium: fibrosis and renin-angiotensin-aldosterone interstitium: fibrosis and renin-ang

14 Becker AE, Caruso G. Congenital heart disease: a morphologist's view on myocardial dysfunction. In: Becker AE, Losekoot TG, Marcelletti C, Anderson RH. eds. PaeAE, Losekoot TG, Marcelletti C, Anderson RH. eds. Paediatric cardiology,

15 Sanchez-Quintana D, Anderson RH, Ho SY. Ventricular myoarchitecture in tetralogy of Fallot. Heart 1996;76:2806.

16 Ohtani O, Ushiki T, Tagauchi T, et al. Collagen fibrillar networks as skeletal frameworks: a demonstration by the cellmaceration/scanning electron microscope method. Arch Histol Cytol 1988;51:249-61.

17 Wang K, Ho SY, Gibson DG, et al. Architecture of atrial muscle in humans. Br Heart $\mathcal{F}$ 1995;73:559-65.

18 Jones CJH, Song GJ, Gibson DG. An echocardiographic assessment of atrial mechanical behaviour. Br Heart $\mathcal{F}$ 1991;65:31-6.
19 Spach MS, Dolber PC. Relating extracellular potentials and their derivatives to anisotropic propagation at a microscopic level in human cardiac muscle: evidence for electrical uncoupling of side-to-side fiber connections with increasing age. Circ Res 1986;58:356-71.

20 Spach MS, Dolber PC, Heidlage JF. Influence of the passive anisotropic properties on directional differences in propagation following modification of the sodium conductance in human atrial muscle: a model of reentry based on anisotropic discontinuous propagation. Circ Res 1988;62:81132 .

21 Thoele DG, Ursell PC, Ho SY, et al. Atrial morphologic features in tricuspid atresia. F Thorac Cardiovasc Surg 1991; 102:606-10.

22 Orie JD, Anderson C, Ettedgui JA, et al. Echocardiographmorphologic correlations in tricuspid atresia. $\mathcal{F}$ Am Coll Cardiol 1995;26:750-8.

23 Carew TE, Cowell JW. Fiber orientation in hypertrophied canine left ventricle. Am F Physiol 1979;236:H487-93.

24 Pearlman ES, Weber KT, Janicki JS, et al. Muscle fiber orientation and connective tissue content in the hypertrophied human heart. Lab Invest 1982;46:158-64.

25 Weber KT, Janicki JS. The heart as a muscle-pump system and the concept of heart failure. Am Heart $\mathcal{F}$ 1979;98:371-

26 Baker EJ, Jones ODH, Joseph MC, et al. Radionuclide measurement of left ventricular ejection fraction in tricuspid atresia. Br Heart $\mathcal{F}$ 1984;52:572-4.

27 Redington AN, Knight B, Oldershow PJ, et al. Left ventricular function in double inlet left ventricle before the Fontan operation: comparison with tricuspid atresia. $\mathrm{Br}$ Heart f 1988;60:324-31.

28 Gibson DG, Traill TA, Brown DJ. Abnormal ventricular junction in patients with univentricular heart. Herz 1979;4: $226-31$

29 Blumenfeld OO, Seifter S. Biochemistry of connective tissue with special emphasis on the heart. In: Robinson TF, Kinne $\mathrm{RKH}$, eds. Cardiac myocyte-connective tissue interactions in health and disease, vol 13. Basel: Karger, 1990:5-36.

30 Ho SY, Jackson M, Kilpatrick L, et al. Fibrous matrix of ventricular myocardium in tricuspid atresia compared with normal heart: a quantitative analysis. Circulation 1996;94: $1642-6$.

31 Lund DD, Twietmeyer TA, Schmid PG, et al. Independent changes in cardiac muscle fibres and connective tissue in rats with spontaneous hypertension, aortic constriction and hypoxia. Cardiovasc Res 1979;13:39-44.

32 Factor SM. Pathological alterations of myocyte-connective tissue interactions in cardiovascular disease. In: Robinson $\mathrm{TF}$, Kinne RKH, eds. Cardiac myocyte-connective tissue interactions in health and disease, vol 13. Basel: Karger, 1990:130-46.

33 Contard F, Koteliansky V, Marotte F, et al. Specific alterations in the distribution of extracellular matrix components within rat myocardium during the development of pressure overload. Lab Invest 1991;64:65-75. 